\title{
Commentary: Does Twitter have a role in improving Family Planning messages and services in Low-and-Middle-Income Countries (LMICs)?
}

\author{
Denise Harrison $^{1 *}$, Saumya RamaRao², Dinesh Vijeyakumar ${ }^{3}$, James McKinnon ${ }^{4}$, Kristina \\ Brown $^{5}$, Stanley Mierzwa ${ }^{6}$ \\ ${ }^{1}$ United States Agency for International Development (USAID), Washington, DC, USA \\ ${ }^{2}$ Population Council, New York City, New York, USA \\ ${ }^{3}$ IBM, Global Health Supply Chain - Procurement and Supply Management (GHSC-PSM) project \\ ${ }^{4}$ Independent Consultant, USA \\ ${ }^{5}$ INOVA, Washington, DC, USA. \\ ${ }^{6}$ Kean University, Center for Cybersecurity, Union, New Jersey, USA
}

\begin{abstract}
Stakeholders are coming together to develop a vision for increasing access to family planning (FP) by 2030. Of the $\underline{923}$ million women in the developing world who wish to avoid a pregnancy, $\underline{218}$ million women are not using a modern contraceptive (Guttmacher Institute, 2020). In 2016, over 3.4 billion people were using the internet (https://ourworldindata.org/internet 2016). Moreover, internet users in the developing world use social media more frequently than Internet users in the U.S. and Europe. Of the many proposed actions to accelerate progress in family planning, the use of Twitter should be a key component.
\end{abstract}

In this commentary, we describe the use of Twitter in a select group of low-and-middle-income countries that have made commitments to the family planning 2020 initiative (FP2020 countries and have the potential to leverage Twitter with current and potential family planning users. We examine Twitter feeds in eight key FP2020 countries, and we look at the content of Tweets issued by the ministries of health in most of these same countries.

Our view is that it is feasible and easy to access Twitter feeds in low-and -middle income countries. We base our view on the types of reproductive health and family planning terms discussed in a public forum such as Twitter by current and potential users and their partners and ministries of health. We highlight two broad considerations that merit discussion among interested stakeholders, including policy makers, program designers, and health advocates. The first relates to the use of Twitter within family planning programs, and the second relates to themes that require more significant research. Data coupled with analytical capacity will help policy makers and program designers to effectively leverage Twitter for expanding the reach of family planning services and influencing social media policy. Our aim is to not only to contribute to the body of knowledge but also to spur greater engagement by program personnel, researchers, health advocates and contraceptive users.

Keywords: Twitter; Public Health; Family Planning; Global Health; Public Health Research;

*Correspondence: deharrison@usaid.gov

DOI: 10.5210/ojphi.v13i2.11094 
This is an Open Access article. Authors own copyright of their articles appearing in the Journal of Public Health Informatics. Readers may copy articles without permission of the copyright owner(s), as long as the author and OJPHI are acknowledged in the copy and the copy is used for educational, not-for-profit purposes.

\section{Introduction}

Sustainable Development Goal (SDG) 3 includes a target "universal access to sexual and reproductive health services including for family planning" https://sdgs.un.org/goals/goal3. Voluntary family planning is a known strategy to reduce maternal and infant mortality and improve the health of women and their children and is considered a "best buy" [1]. Today, stakeholders are coming together to develop a vision for increasing access to family planning by 2030 by influencing social systems and supporting data-informed decisions to accelerate progress. Social media, including Twitter, could be a key component for social influence and data generation and analysis. Twitter is one of the most popular forms of microblogging. Unlike other social media platforms such as Facebook, Instagram, or Whatsapp, Twitter posts are generally public and hence can be collected and analyzed. We feel there is a growing sense in the field of international family planning that social media and digital forms of communication will be able to reach health consumers more efficiently and help family planning programmers and advocates respond to their needs. Family planning (FP) programs should comprehensively incorporate Twitter to communicate to potential and current FP users.

In this commentary, we describe the use of Twitter in eight (Bangladesh, India, Indonesia, Ghana, Liberia, Kenya, Nigeria, and South Africa low-and-middle-income countries (LMICs)) and discuss the potential to leverage Twitter for more engagement with current and potential users of family planning.

\section{What are people talking about?}

We examine Twitter feeds in two different ways at various points in times in eight key FP2020 countries. First, we look at Tweets found in country Twitter feeds for a one-week period in 2016 (Jan $1^{\text {st }}$ to $\left.8^{\text {th }}\right)$ and in 2020 (Nov $1^{\text {st }}$ to $\left.10^{\text {th }}\right)$. 


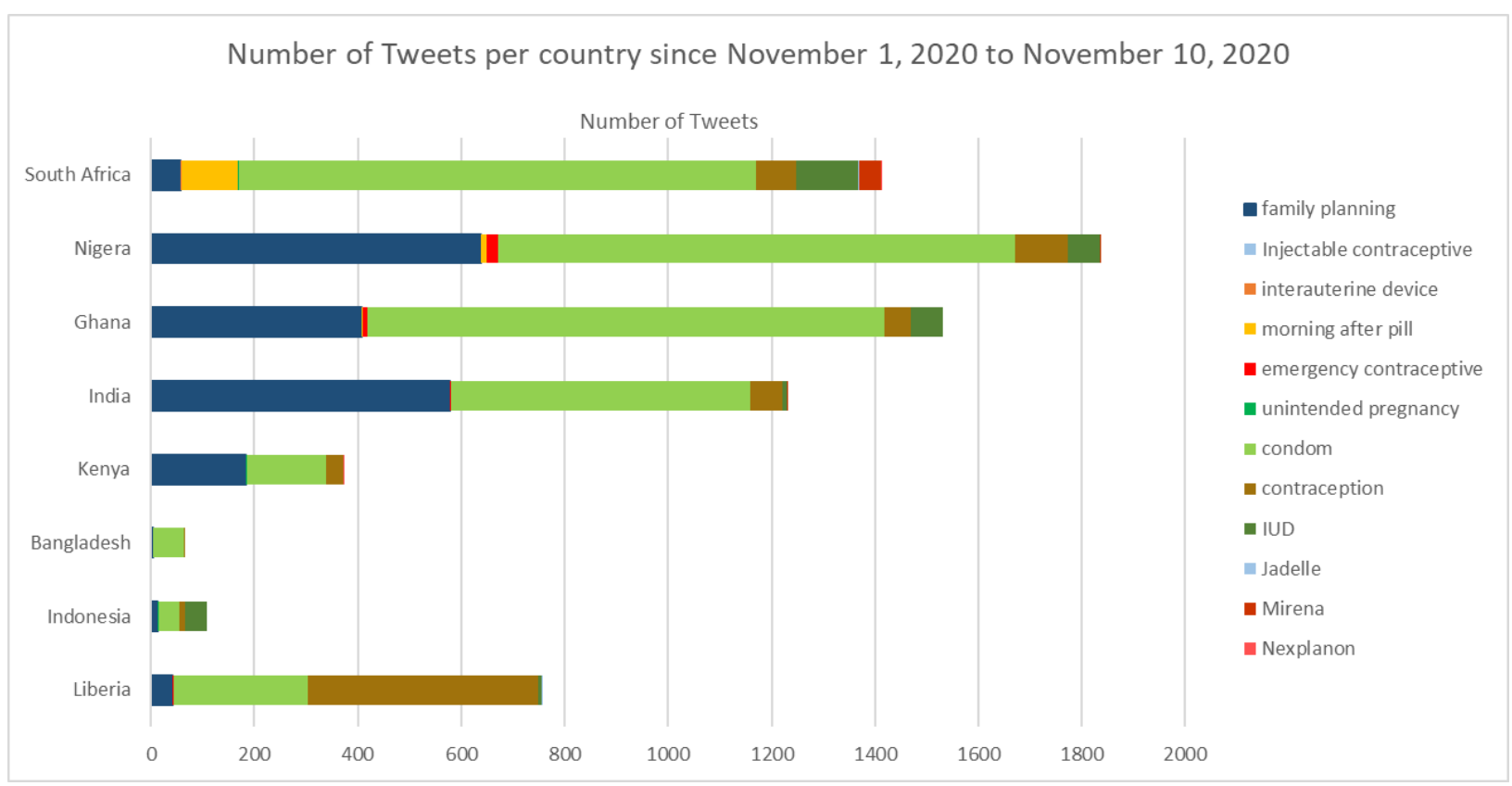

Figure 1: Tweet summary by keywords for the period November 1-10, 2020 (Tweets below 100 count, not visible in the chart)

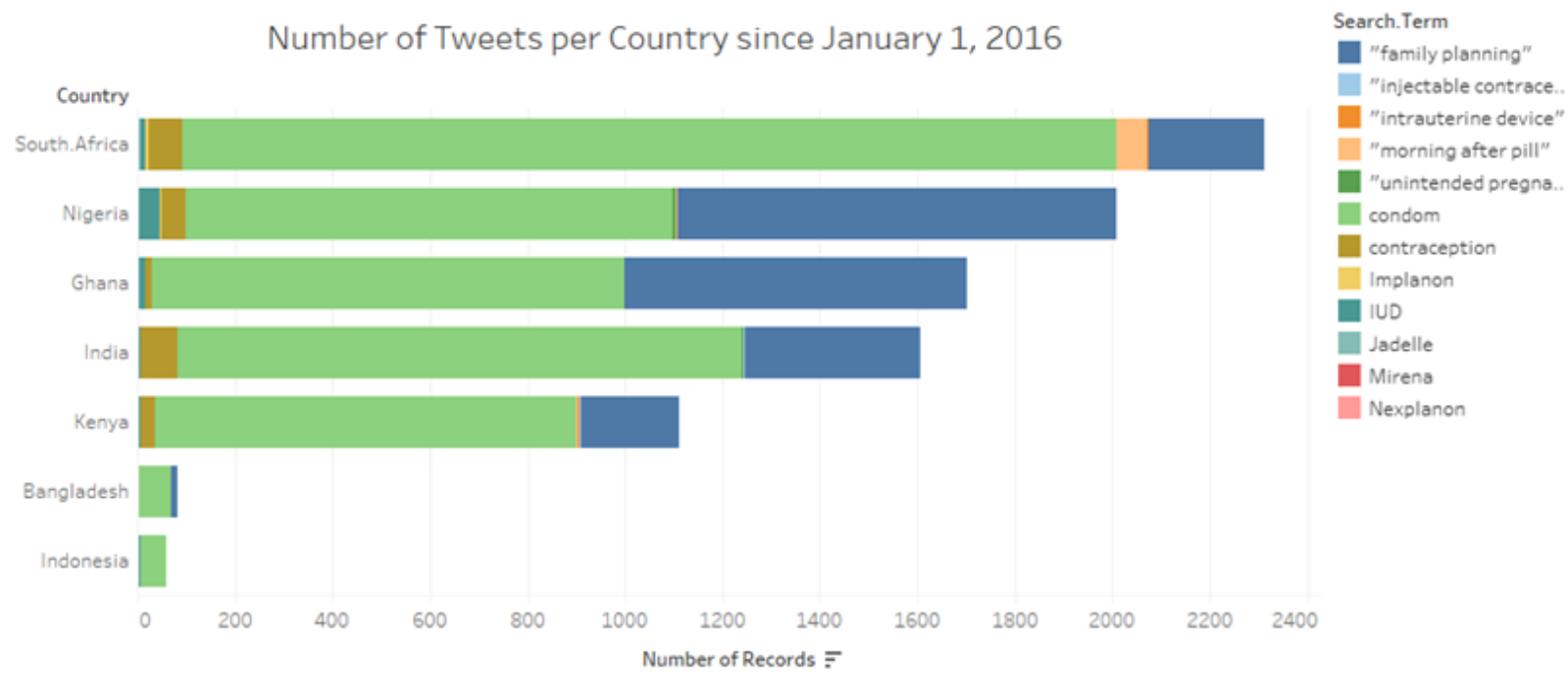

Figure 2: Tweet summary by key words for the period November 1- 10, 2020 (Tweets below 100 count, not visible in the chart)

In all countries, there were public tweets on the issue of family planning. Countries such as South Africa, India, and Nigeria had a vibrant and active Twitter conversation while others such as Liberia, Kenya and Ghana, Indonesia, and Bangladesh had tweets but were not substantial. 
We believe that many discussions on social media were occurring in languages other than English.

We noted that some words such as Condom and family planning were tweeted frequently while the intrauterine device (IUD) was rarely used. The mention of specific contraceptives is in line with our expectation that only those that are widely used or are new to the market will likely be discussed on social media. In terms of actual methods discussed, IUD use is one of the least discussed methods among the methods discussed; interestingly, we also find that "IUD" is more commonly used than "intrauterine device" indicating fluency in the general population about this term. While there might be fluency about the term, it is difficult to ascertain the depth of knowledge about IUDs.

We found that condoms were discussed more than any other method. We speculate that this discussion could have been spurred due to greater concern about protection and prevention of HIV and STIs since the tweets themselves do not make clear as to the underlying motivation, but we see a high amount of HIV/condom discussion in South Africa, which has a high HIV prevalence rate. Relatedly, myths around condoms and their ability to protect against HIV were found in a group of tweets on condoms in Nigeria and were retweeted many times in 2016. During the second analysis in 2020, there were a lot more tweets related to IUDs from non-governmental organizations (NGOs), civil society organizations (CSOs), advocates and well as manufacturers. One tweet, "Wahala for women wey get IUD o" from @debo_bohor based in Nigeria, complaining about the difficulty women have obtaining IUDs, was retweeted over 50 times in Nigeria and neighboring Ghana.

Third, we found myths and misconceptions and an effort to dispel them as well. Myths around condoms and HIV were also detected in the script, such as the belief that withdrawal prevents HIV transmission. Tweets around this myth were repeated and pervasive during the first round of analyses from 2016. However, in 2020, many NGO, civil society organizations (CSO), and government workers sent tweets countering myths and explaining how different methods work, especially condoms, IUDs, and Emergency Contraception (EC). In Nigeria, @Chief_ozomma, based in Abuja, Nigeria, responsible for logistics, retweeted: @onelifeng: "Withdrawal method is one of the least effective methods of all the methods of contraception. It is a recommendation to make use of condoms during sexual intercourse not only to prevent pregnancy but to also prevent STIs." Onelife Initiative for Human Development is an Ibadan-based civil society organization focused on youth and sexual and reproductive health. We used the global guidance on family planning provided by the WHO to verify the validity of the tweets.

Fourth, we noted that although there appears to be general discussion about contraception and family planning, it tends to be less about specific brands. However, there was more product and service promotion by manufacturers and social marketing organizations during November 2020 than in 2016, indicating that market players could be using Twitter to build demand for their products. Also, hashtags were not found as often as search terms on their own, indicating there are not a lot of campaigns around contraception on Twitter in these countries. When specific contraceptives are discussed, not all methods are discussed equally.

Finally, we learned that the media (newspaper/television) assist in family planning messaging on Twitter. For example, in the 2020 feed, an analysis of popular users (tweets with most likes) found 
that the tweets originated from the media. This suggests that Twitter, combined with conventional media, can amplify family planning messaging and discussion.

\section{What do Ministries of Health tweet about?}

On reviewing tweets issued by national ministries of health during May 25 - June 24, 2019 as well as between May 14 - August 17, 2020, the majority were on ceremonial events, recounting the news of government officials and some policy announcements. Some ministries, notably the Ministry of Health and Family Welfare (MoHFW) in India sent primarily educational tweets. The $\mathrm{MoH}$ of Kenya had many tweets about preventing Ebola and surveillance updates last year. None of the ministries of health followed many people or organizations. However, the number of followers grew exponentially from 2019 to 2020, indicating that people are following the MOH more on Twitter.

Only the ministries of health of Kenya and India had tweets on family planning during the 30-day period of May 25 - June 24, 2019. @MOH_Kenya also tweeted advice against pre-marital sex, especially for teens. @MoHFW_India issued nearly 20 tweets on menstrual hygiene management in recognition of the celebration of World Menstrual Hygiene Day.

\section{What does this mean for family planning?}

Based upon our exploration of the potential of harnessing Twitter for FP programming, we identified two broad considerations that merit discussion among interested stakeholders including policy makers, program designers and health advocates. The first relates to the use of Twitter within family planning programs for program enhancement and the second relates to themes that require greater research and analysis. By program enhancement, we mean improved reach of family planning programs to educate and orient users and potential users resulting in a more informed clientele.

Program enhancement: We foresee the potential for including analysis of tweets for message development and program planning and say this for two reasons. First, we anticipate that there will continue to be growth in the use of social media for discussion on family planning and related health issues. Second, as adeptness in social media use increases and new forms of community conversation become commonplace, we can anticipate that consumers of family planning and health care will be engaging with the health system in different ways. Ministries of health, NGOs, and CSOs need to be ready to respond to user concerns and community chatter to design effective programs. They also need to understand Twitter users' and their networks to positively influence the conversation around family planning access and use. This implies that program designers need to plan for modern communication strategies that do not rely exclusively on traditional models of information education communication (IEC). It implies that family planning programs need to include tech and media-savvy communication teams on their roster to be able to respond in realtime to user needs and priorities for engagement with the family planning program.

We believe that there is potential for program managers and clinic personnel to learn about the "buzz outside the clinic," especially for youth [2] who are using Twitter more frequently, so that they can appropriately design and tailor their services and products to serve the needs of their populations. We acknowledge that program personnel may not get a comprehensive understanding 
of the issues or underlying and less explicit concerns that consumers might have; nevertheless, they will be able to get a better sense of the range of consumer concerns and fears than they might learn in a formal provider-consumer interaction during service provision.

We provide four examples of the ways in which family planning programs can employ Twitter to engage with consumers. The first example is in dispelling myths. One predominant area is in timely myth-busting especially given the speed at which rumors and false information can spread. We anticipate that there is potential for Twitter to dispel myths around family planning, as it has done for Ebola, and is doing for COVID-19 [3]. For example, the anti-vaccine campaign in the US worked through social media platforms, including Twitter during the measles outbreak in 2015 [4] and more recently in 2019. Although there were responses from official health agencies such as the Centers for Disease Control (CDC) as well as mainstream media outlets, the narrative was led by grassroots campaigners. The lessons learnt from episodes such as this and the handling of the Ebola epidemic provide direction on how official health agencies need to coordinate with media to counter myths and provide accurate, evidence-based information.

A second way that programs can use Twitter is for improving existing messaging about contraceptive supply chains and services and likely direct users to points of care, whether in the public or private sectors. The Kenyan $\mathrm{MoH}$ used Twitter to announce that there were sufficient supplies of antibiotics during the studied 30-day period and could use Twitter to announce the availability of FP supplies, as could other ministries of health. Many contraceptives are being introduced or being offered through multiple service outlets - these include contraceptive vaginal rings, the Sayana ${ }^{\circledR}$ Press injectable available through many types of providers, including for selfadministration, and various hormonal intra-uterine systems. It will be necessary to support the rollout of these contraceptives with messaging aimed at consumers to make them aware of the product, the different types of outlets to seek information and services, as well as provide support in the use of the chosen contraceptive. Finally, as we have seen during the COVID-19 pandemic, some family planning programs have offered consultations through telemedicine; Twitter can be used to inform health consumers of such service options if they are pertinent to that program setting.

The third area for program enhancement is around social and behavior change communication. Twitter might serve as a modern tool for promoting healthy behaviors by addressing stereotypes, increasing awareness of family planning options and creating a community of users; for example, linking people to websites and organizations that have accurate information on family planning and promoting a community of family planning users. Similarly, having influencers tweet about their healthy habits might serve as an endorsement for their followers to adopt similar habits.

A fourth area is around accountability for supplies. While using closed Facebook pages to report FP stock-outs at the facility level occurs in some countries such as the Philippines (see https://www.facebook.com/groups/commoditymonitoring/). Twitter could be used as an open and transparent mechanism for accountability. Women could let neighbors and government officials know when there are stock-outs at their local service delivery point. This is similar to airline passengers directly tweeting to the airline company the problems or grievances they have in their travel. Relatedly, government officials and neighbors could let women know when new stocks or new methods are available at local service delivery points. Twitter could also be used among health 
workers to transmit more detailed types of data - such as pictures of monthly stock reports and orders.

Research: In the domain of research, there is much to be learned. There is a body of research largely led by computational scientists on scanning and analyzing tweets to measure the saliency of the topic being discussed and the fervor behind the topic-in other words, the sentiment and buzz. It is intuitive and to be expected that interest in a particular topic swells in response to an event, milestone or ongoing crisis. For example, 10.5 million tweets containing the word "Ebola" were sent from 170 countries between September 16 and October 6, 2014 during the time the epidemic was occurring [4].

We believe that there is an audience interested in family planning and sentiment analysis [5] could be conducted. With the increasing saturation of internet access, we anticipate that family planning will be discussed more. Since Twitter is a low cost and viable tool, it will be possible to use it for monitoring and assessing family planning attitudes and interests. Concomitantly, it will be necessary to train family planning program managers in sentiment analysis and buzz so that they can shape interventions and develop messaging in response to Twitter feed.

A second area of research is that not much is known about the coverage of Twitter, how and how well messages through Twitter work to bring about social and behavior change, especially in matters related to family planning and reproductive health. We know from other health areas such as cancer prevention have used Twitter for health messaging that some changes in attitude and knowledge are possible but no evidence of behavior change [6].

The extent to which the potential of Twitter analysis can be harnessed for program design will depend on the ease with which the analysis can be done by the staff on the roster with social media abilities. Possible ways to facilitate the process exist. For example, the open-source R package Shiny allows users to easily build interactive web apps that can be standalone on a webpage or embedded, could be developed for governments, especially local government, civil society, NGOs, and donors to facilitate following Twitter feeds. A Shiny app used weekly by programmers could inform FP programs and policies on a real-time, low-cost basis. Shiny is a good option for fast prototyping and requires less programming capacity than other frameworks that could accomplish the same goal. For example, Java is not as user-friendly for non-programmers, and development of a simple GUI app can take time.

\section{Call to action}

Our view is that it is feasible to use Twitter in LMIC countries because there is interest in public discussion about family planning. It is no longer a topic meant solely for discussion privately within families or between consumers and health care providers. Moreover, two-thirds of the world uses the internet, and new internet and social media users are growing at a faster pace in many FP2020 than in the U.S. and Europe. Many people access the internet through smartphones instead of computers. In 2015, smartphone ownership in the developing world grew 37\% annually [7].

We believe that there is potential for the use of Twitter as a communications tool that family planning programs in LMICs can use. We also caution that before the wide-spread use of Twitter, health programmers conduct cybersecurity assessments to identify any potential weakness and 
develop mitigation plans to counter the threat should they occur [8]. Finally, we also call for research from many program settings and geographical regions to assess practical and realistic applications. As greater fluency in the use of Twitter among program planners and health consumers increases, we anticipate different media strategies will be required for each country. By doing so, family planning programs will be better prepared to serve a new generation of users who are socialized to communicate in new mediums than their predecessors. Progress towards SDG 3 will thus be made easier by harnessing the potential of Twitter to serve larger segments of the population.

\section{Acknowledgments}

This research study and report was supported by the Packard Foundation under a grant to the Population Council.

\section{References}

1. Starbird E, Norton M, Marcus R. 2016. Investing in family planning: Key to achieving the Sustainable Development Goals. Glob Health Sci Pract. 4(2), 191-210. PubMed https://doi.org/10.9745/GHSP-D-15-00374

2. Pew Research Center, June, 2018, "Social Media Use Continues To Rise in Developing Countries, but Plateaus Across Developed Ones"

3. Limaye RJ, Sauer M, Ali J, Bernstein J, Wahl B, et al. 2020. Building Trust while influencing online COVID-19 content in the social media world. Lancet Digit Health. 2(6), E277-78. doi:https://doi.org/10.1016/S2589-7500(20)30084-4. PubMed

4. Luckerson V. 2014. Watch how word of Ebola exploded in America. https://time.com/3478452/ebola-Twitter/.

5. Rosenthal S, Farra N, Nakov P. 2017. Sentiment analysis in Twitter. Proceedings of the 11th International Workshop on Semantic Evaluations (SemEval-2017), pages 502-518, Vancouver, Canada, August 3 - 4, 2017. Association for Computational Linguistics.

6. Gough A, Hunter RF, Ajao O, Jurek A, McKeown G, et al. 2017. Tweet for behavior change: Using social media for the dissemination of public health messages. JMIR Public Health Surveill. 3(1), e14. PubMed https://doi.org/10.2196/publichealth.6313

7. Pew Research Center. "Smartphone Ownership and Internet Usage Continues to Climb in Emerging Economies.” February 2016 http://www.pewglobal.org/2016/02/22/smartphoneownership-and-internet-usage-continues-to-climb-in-emerging-economies/

8. Mierzwa, S. RamaRao, S, Yun, JA. and Jeong, BG. 2020. Proposal for the Development and Addition of a Cybersecurity Assessment Section into Technology Involving Global Public Health. International Journal of Cybersecurity Intelligence \& Cybercrime. 3(2), 48-61. https://doi.org/10.52306/03020420BABW2272 